10 years ESJ

Special edition

\title{
Nueva Normalidad en las Micro Unidades Económicas Turísticas de la Sierra Norte de Puebla
}

\author{
Ana Luisa Gamboa Gochis \\ Edgar Hernández Zavala \\ Universidad el Valle de Puebla, Mexico \\ Anaid Guerra García \\ Benemérita Universidad Autónoma de Puebla, Mexico
}

Doi: $10.19044 /$ esj.2021.v17n4p259

Submitted: 28 October 2020

Accepted: 29 December 2020

Published: 07 February 2021

\author{
Copyright 2021 Author(s) \\ Under Creative Commons BY-NC-ND \\ 4.0 OPEN ACCESS
}

Cite As:

Gamboa Gochis L.A., Zavala H.E. \& García G.A. (2021). Nueva Normalidad en las Micro Unidades Económicas Turísticas de la Sierra Norte de Puebla. European Scientific Journal, ESJ, 17(4), 259. https://doi.org/10.19044/esj.2021.v17n4p259

\section{Resumen}

La actividad turística, una de las más dañadas en la Sierra Norte del Estado de Puebla (México) por la crisis del coronavirus COVID-19 provocó el cierre parcial o total de decenas de Micro Unidades Económicas (MUE), poblaciones como Cuetzalan del Progreso y Chignahuapan donde se realizó el presente estudio, reconocen que deberán adaptarse a las nuevas circunstancias, tanto en espacio, capacitación y difusión para mantener su presencia en los consumidores a través de sus servicios; de ahí que los giros empresariales como Hoteles, Tours, Restaurantes entre otros, deberán mantener una comunicación continua, información oportuna y adaptarse rápidamente a las normas que se dicten oficialmente en la materia con el fin de reinsertarlos a la dinámica económica de la región. Se realizó un análisis metodológico mixto de UE con giros turísticos, de donde se identificaron factores organizacionales externos, internos y de comunicación, cuyos resultados reflejaron numerosas áreas de oportunidad para una toma la de decisiones para diseñar y proponer estrategias de mercadotecnia, adaptadas a las tendencias internacionales que se toman debido a la afectación del SARS CoV2. 
Palabras clave: MUE turísticas, planeación estratégica, nueva normalidad empresarial

\title{
New Normality in the Micro Tourist Economic Units of the Sierra Norte de Puebla
}

\author{
Ana Luisa Gamboa Gochis \\ Edgar Hernández Zavala \\ Universidad el Valle de Puebla, Mexico \\ Anaid Guerra García \\ Benemérita Universidad Autónoma de Puebla, Mexico
}

\begin{abstract}
The COVID-19 crisis affected a lot of activities in the Sierra Norte of the State of Puebla (Mexico) including tourism activity. This resulted to the partial or total closure of dozens of Micro Economic Units (EUTM). This study was carried out in Cuetzalan del Progreso and Chignahuapan and they realised the need to adapt to the new circumstances both in space, training, and dissemination to maintain their consumers through their services. For this reason, business tours such as hotels and restaurants must maintain a continuous communication, timely information, and adapt quickly to the rules that are officially dictated in this field in order to reinsert them to the economic dynamics of the region. A mixed EU methodological analysis was carried out from which external, internal, and communication organizational factors were identified. This resulted to numerous areas of opportunity for decision-making to design and propose marketing strategies, which are adapted to international trends that are taken due to the involvement of SARS CoV2.
\end{abstract}

Keywords: Tourist (EUTM), strategic planning, new business normality

\section{Introduction}

El estado de Puebla, México se localiza en la zona centro sur del país y lo integran 217 municipios. Se divide en siete regiones, entre las que destaca la Sierra Norte caracterizada por sus múltiples atractivos de naturaleza y diversidad cultural que son foco de la atención para turistas nacionales y extranjeros.

El presente estudio utilizó como referencia la base de datos de la DENUE $^{1} 2019$, para los municipios de Chignahuapan y Cuetzalan ubicados en la anterior demarcación señalada y captan el mayor flujo e interés de los

\footnotetext{
${ }^{1}$ Dirección Estadístico Nacional de Unidades Económicas
} 
visitantes a la zona. De esas estadísticas, se identificó al grupo de MIPYMES de giro turístico, que por su número y tamaño (entre 1 y 10 trabajadores), son consideradas Micro Unidades Económicas (MUE).

El número de MUE turísticas en ambos municipios serranos es de 190 establecimientos, de donde la visita turística nacional e internacional a estas poblaciones se calculó en 102432 personas en 2017 (INEGI, 2017) y disminuyó severamente en un 70\% apenas en marzo de 2020 (Según apreciación de las Presidencias Municipales respectivamente) ante la situación de salud que se presenta a nivel mundial, lo que afectó a la derrama económica en un porcentaje sin precedentes.

Este trabajo, por lo tanto, tiene la finalidad de sugerir factores de competitividad y de marketing estratégicos que permitan detonar la captación de clientes por las MUE turísticas, como una alterativa de apoyo ante los retos que impone la nueva realidad.

Los efectos de la retracción del turismo se sentirán en particular en las micro y pequeñas empresas, cuyo peso en el sector de hoteles y restaurantes es enorme: el 99\% de las empresas y el 77\% del empleo (CEPAL, 2020). En este proyecto se plantean posturas teóricas que tienen implicaciones directas ante la realidad que las empresas pueden aplicar en cuanto a estrategias de marketing. Como tal, al identificar las necesidades de los consumidores, se plantean estrategias orientadas a cubrir dichas necesidades ante la situación de pandemia. Es por eso que el turista deberá ajustarse a los nuevos lineamientos protocolarios dictaminados en la SECTUR (2020) donde se "establecen las recomendaciones para el sector turístico, enfocadas en prevenir y mitigar al máximo los riegos de contagio mediante medidas de higiene personal, del entorno y de sana distancia, como una prioridad en la nueva normalidad, tomando como base el semáforo por regiones, publicado en el Diario Oficial de la Federación".

Debido a las nuevas circunstancias, las estrategias de mercadotecnia pueden desarrollarse en diversos niveles: para la venta, la publicidad, los servicios al cliente, la administración de producto e investigación de mercados. Esta relación se fundamentará en las 4ps del marketing (producto, precio, plaza, promoción); propuesto por E. Jerome McCarthy (1960) consideradas como parte del marketing clásico.

Posteriormente, se propone una segunda generación de las $4 \mathrm{ps}$ propuesta por Idris Motee (2001): (personalización, participación, par a par para las comunidades, y predicciones modeladas) de enfoque digital. Otro fundamento teórico se apoyará en las postulaciones de Michael Porter (2015), como referente en el sector empresarial con respecto las cinco fuerzas donde plantea estrategias corporativas para fortalecer la rentabilidad a partir del diagnóstico de la empresa para identificar aspectos fiables de fortalecimiento para el mercado al que va dirigido. 
De dicho autor, se toma como referente el modelo de las cinco fuerzas que identificará la rentabilidad de la empresa a través de:

1.- Competidores potenciales: Identificará que es lo que ofrece la competencia para generar estrategias.

2.- Poder de negociación con proveedores: A través de estrategias negociará con ellos para mantenerlos.

3.- Poder de negociación de los compradores: Se generarán estrategias para afrontar situaciones de riesgo del producto.

4.-Rivalidad entre competidores actuales: Estrategias que permitan aprovechar las fortalezas de la empresa.

5.- Riesgo de sustitución: Estrategias que impidan el consumo de otro producto similar.

Otro aspecto necesario para analizar en el mercado, es el desarrollo de cadenas productivas en el turismo, las cuales deben fortalecerse como actividad económica transversal. Esta acción se había propuesto como parte de la visión 2040, en el Foro de turismo es bienestar (SECTUR ${ }^{2}$, 2018) sobre todo a las micro, pequeñas y medianas empresas, donde se requiere la alineación de autoridades, agentes económicos y productivos (p.39); como resultado de ello se fortalecen los medios de comunicación sustentada en las TIC, somo una manera en que las empresas pueden operar, ya que se comunican, planean y analizan la forma de mejorar con eficiencia la rentabilidad de los negocios del sector. En estos canales es donde se espera la integración de la oferta con la demanda y el financiamiento para fortalecer a las MUE a bajo costo, mejorando la infraestructura logística, que se traduzca en costos competitivos dentro del mercado del turismo global.

\section{Método}

El estudio aplicado a las MUE turísticas constó de 3 instrumentos: cuestionario, formulario y entrevista. El cuestionario, se aplicó con el fin de identificar a los clientes de los negocios y generar su ficha técnica correspondiente, ubicar los factores que influyen en la toma de decisiones dividido en tres ejes que se consideran como factores de competitividad, así como las características de los perfiles de servicio. Ver Tabla 1.

\footnotetext{
${ }^{2}$ Secretaría de Turismo
} 
Tabla 1. Factores de competitividad

EJE 1

\begin{tabular}{|c|c|c|}
\hline $\begin{array}{l}\text { Factores externos: } \text { Aspectos } \\
\text { con los que la }(M U E) \text { debe } \\
\text { relacionarse para ser } \\
\text { competitiva }\end{array}$ & $\begin{array}{c}\text { Factores internos: Elementos } \\
\text { que están dentro de la (MUE) y } \\
\text { pueden ser controlados }\end{array}$ & $\begin{array}{l}\text { Comunicación Empresarial: } \\
\text { Manera de transmitir } \\
\text { información precisa al personal, } \\
\text { al entorno y al cliente }\end{array}$ \\
\hline $\begin{array}{lll}\text { - } & \begin{array}{l}\text { Contexto de } \\
\text { empresa }\end{array} & \text { la }\end{array}$ & $\begin{array}{l}\text { - Misión, Visión y } \\
\text { Valores }\end{array}$ & - Toma de decisiones \\
\hline $\begin{array}{l}\text { Pertenencia a un } \\
\text { organismo }\end{array}$ & - Organigrama & - Comunicación interna \\
\hline - $\quad$ Precio del producto & $\begin{array}{l}\text { - Número y Antigüedad } \\
\text { de empleados }\end{array}$ & $\begin{array}{l}\text { - Comunicación externa y } \\
\text { venta de su producto y/o } \\
\text { servicio }\end{array}$ \\
\hline - $\quad$ Target & $\begin{array}{l}\text { - Número en genero de } \\
\text { empleados }\end{array}$ & - Eficiencia en el servicio \\
\hline $\begin{array}{l}\text { - Tipo de servicio o } \\
\text { producto }\end{array}$ & $\begin{array}{l}\text { - Prestaciones, } \\
\text { Incentivos y } \\
\text { compromiso laboral }\end{array}$ & - Atención al cliente \\
\hline - Sucursales existentes & $\begin{array}{l}\text { - Eficiencia } \\
\text { seguimiento laboral }\end{array}$ & - Identificador visual \\
\hline
\end{tabular}
Fuente: Elaboración Propia

\section{EJE3}

municación Empresarial mación precisa al personal, al entorno y al cliente

- Toma de decisiones

- Comunicación interna

Comunicación externa y venta de su producto $\mathrm{y} / \mathrm{o}$

Número en genero de

Prestaciones, Incentivos $\quad \mathrm{y} \quad \bullet \quad$ Atención al cliente Eficiencia $\quad \mathrm{y}$ seguimiento laboral $\quad$ Identificador visua

El formulario, se aplicó para identificar la afectación directa del COVID 19 en la (MUE), con base en los siguientes aspectos vinculados de los efectos de la enfermedad. Ver Tabla 2.

Tabla 2. Factores de afectación

EJE 1

Factores externos:

Consecuencias de afectación directa a la (MUE)
EJE 2 Factores internos: Afectaciones internas a la (MUE)

EJE3

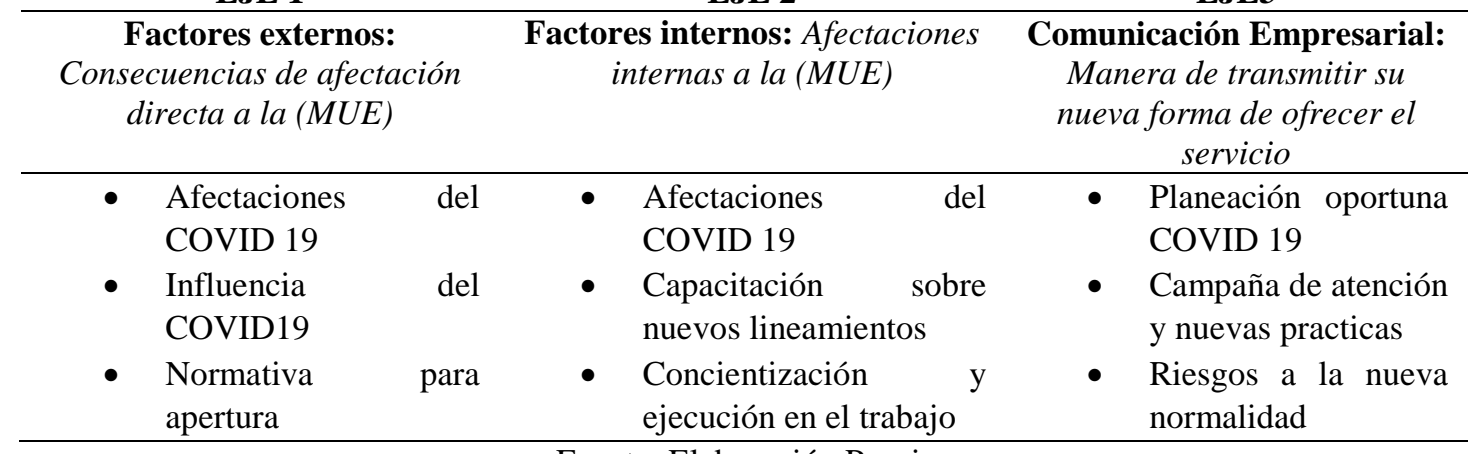

Fuente: Elaboración Propia

Las entrevistas, se realizaron por medio de una videoconferencia para conocer de parte de los dueños de las (MUE) su toma de decisiones y estrategia (FODA). Ver Tabla 3. 
Tabla 3. Análisis estratégico (FODA)

\begin{tabular}{l|l} 
FORTALEZAS (Mantenerlas) & DEBILIDADES (Corregirlas) \\
\hline OPORTUNIDADES (Explotarlas) & AMENAZAS (Afrontarlas) \\
Fuente: Elaboración Propia
\end{tabular}

\section{Hallazgos}

Derivado de los instrumentos de investigación aplicados a factores decisivos en las actividades turísticas, se obtuvo información directamente de los Micro Empresarios en el marco de la pandemia por COVID-19, así como también de las acciones planteadas por el gobierno del estado a través de la dirección de Turismo de Cuetzalan y Chignahuapan, ante la necesaria reactivación social por su importancia económica para la región se destacó " 5 lineamientos de Atención y Buenas Prácticas" para llevarlas a cabo por las (MUE) que ofrecen servicios turísticos en esta zona.

No obstante, la situación de riesgo sanitario y la iniciativa de las direcciones de Turismo Municipal de ambos Ayuntamientos, sólo el 50\% de los dueños de negocios dice conocer los nuevos lineamientos protocolarios para la reapertura indicados por la Dirección del Turismo Municipal, ya que explican que sólo algunos tomaron la capacitación necesaria para su personal, mientras que los otros se enteraron un poco de dicha información por terceras fuentes.

El instrumento aplicado en esta investigación para preguntar acerca de la afectación de la enfermedad del COVID19 a las MUE, indicó que ésta fue del $75 \%$ directamente a sus ingresos debido al cierre parcial o total de sus establecimientos. Ante la situación del confinamiento el 50\% tuvo que hacer recorte de su personal, el $25 \%$ cierre parcial y el $25 \%$ cierre total. Es curioso que una cuarta parte de los entrevistados define esta pandemia como una oportunidad, ya que argumentan que el turista ahora busca estar en mayor contacto con la naturaleza; dicha respuesta fue dada especialmente por las MUE cuya ubicación se encuentra fuera de las poblaciones semi urbanas.

A continuación, se describen e identifican los factores de competitividad y perfil de las empresas turísticas de ambos municipios obtenidas en las entrevistas a empresas turísticas. Primero, se resalta que un $75 \%$ son MUE con por lo menos 5 años de presencia en el mercado y el $25 \%$ dice tener más de 20 años de haberse fundado y los entrevistados dicen no contar con sucursales y muy pocas están adheridas a un organismo indígena denominado "Masehual Siuamej Mosenyolchicauani S. de S.S." que cuenta con Misión, Visión y Valores, así como un organigrama gremial. Cabe destacar que el $66.7 \%$ de las Unidades Económicas ofrece prestaciones laborales al personal debido a que están inscritas en el IMSS.

Por otra parte, se destaca que como productos principales se ofertan el hospedaje $75 \%$ y los alimentos en restaurante el $25 \%$. Los precios los definen 
los dueños $66.7 \%$ sin considerar alguna técnica específica de costeo y dan a conocer sus servicios y/o productos a través de venta directa $40 \%$, campaña publicitaria $40 \%$ y en sus locales $20 \%$ destacando la mayoría de las veces por sus precios bajos. En tal sentido, las campañas según su argumento (de los empresarios) en un $80 \%$ se dirige a un mercado general y un $20 \%$ al segmento familiar. No hay un enfoque de género específico.

Los dueños de MUE comentan que incentivan a sus clientes por medio de precios bajos en un $80 \%$ y en segundo término la calidad del producto y servicio, al cual identifican preguntándole directamente al cliente en un $60 \%$ de las veces o por medio de una breve encuesta. Se ratifica que es recurrente que la comunicación publicitaria se haga por medio de redes sociales en un $80 \%$ por medio de Facebook, Instagram y Twitter principalmente, no obstante que apenas un $80 \%$ cuenta con una imagen (logotipo) que identifica a su MUE. En ese tenor, el $50 \%$ de los dueños considera que se requerirá reforzar la estrategia o realizar ajustes a la imagen. Es notable que el $75 \%$ indica que su empresa se adecuará a las nuevas tendencias sanitarias y de mercado, mientras que el 25\% cree que sólo alguna de sus áreas cambiará.

En lo referente a la planeación estratégica, solo el 50\% de los dueños dice considerarla necesaria en la organización y en la mercadotecnia para fortalecer el posicionamiento e imagen de la empresa. Los que dicen conocerla, buscarán medios adecuados para llegar a su nicho de mercado turístico anterior o a nuevos clientes que coinciden con la búsqueda de espacios naturales al aire libre en medio de la pandemia. El 25\% expresa que busca asesorías de expertos y se capacita en esas áreas empresariales.

Con respecto a las entrevistas realizadas virtualmente con los dueños de MUE, se llegó a un primer planteamiento de un análisis estratégico de Fortalezas, Oportunidades, Debilidades y Amenazas (FODA), ya que se trabaja frecuentemente la manera de reorganizarse para mejorar su servicio. Los dueños dicen que tienen profundas debilidades porque en su mayoría son micro empresas, sin embargo, reconocen que es necesario el apoyo de expertos que ojalá fueran impulsados por las autoridades turísticas.

El consenso generalizado es que se tienen fortalezas en el precio y el servicio al cliente, y las debilidades son no contar con servicio de restaurante en la mayoría de hoteles, considerando este factor como una oportunidad a futuro, mientras que consideran como amenaza la falta de capacitación y comunicación con la autoridad.

\section{Conclusiones}

Durante la pandemia de COVID-19, las MUE experimentaron una fuerte desaceleración colocándolas en una muy alta vulnerabilidad económica. La nueva realidad genera el planteamiento de nuevos lineamientos basados en las disposiciones de las instancias de gobierno a través de los municipios y sus 
direcciones de Turismo que se deben propagar por diversos medios como protocolos de acción para la reapertura de sus actividades.

Es importante mencionar que, dadas estas disposiciones, no se ha coordinado realmente un solo esfuerzo y cada población genera su propio control de reaperturas en lo referente a los servicios turísticos, sin embargo, se destaca que el medio con el que se difunden las nuevas disposiciones es la página oficial de cada ayuntamiento, así como por redes sociales.

Cabe destacar que estas experiencias llevan a una reflexión acerca de lo indispensable que es la intervención de las autoridades en sus distintos niveles que conlleven a coordinar una reapertura y adaptación paulatina a nuevas circunstancias que la salud y el consumidor demandan, sin embargo, es importante reformular que el análisis generó variables competitivas que desde un inicio no se consideraron; ahora reflejan acción importante como parte de la estrategia de marketing.

\section{Factores, competitividad y de marketing estratégico}

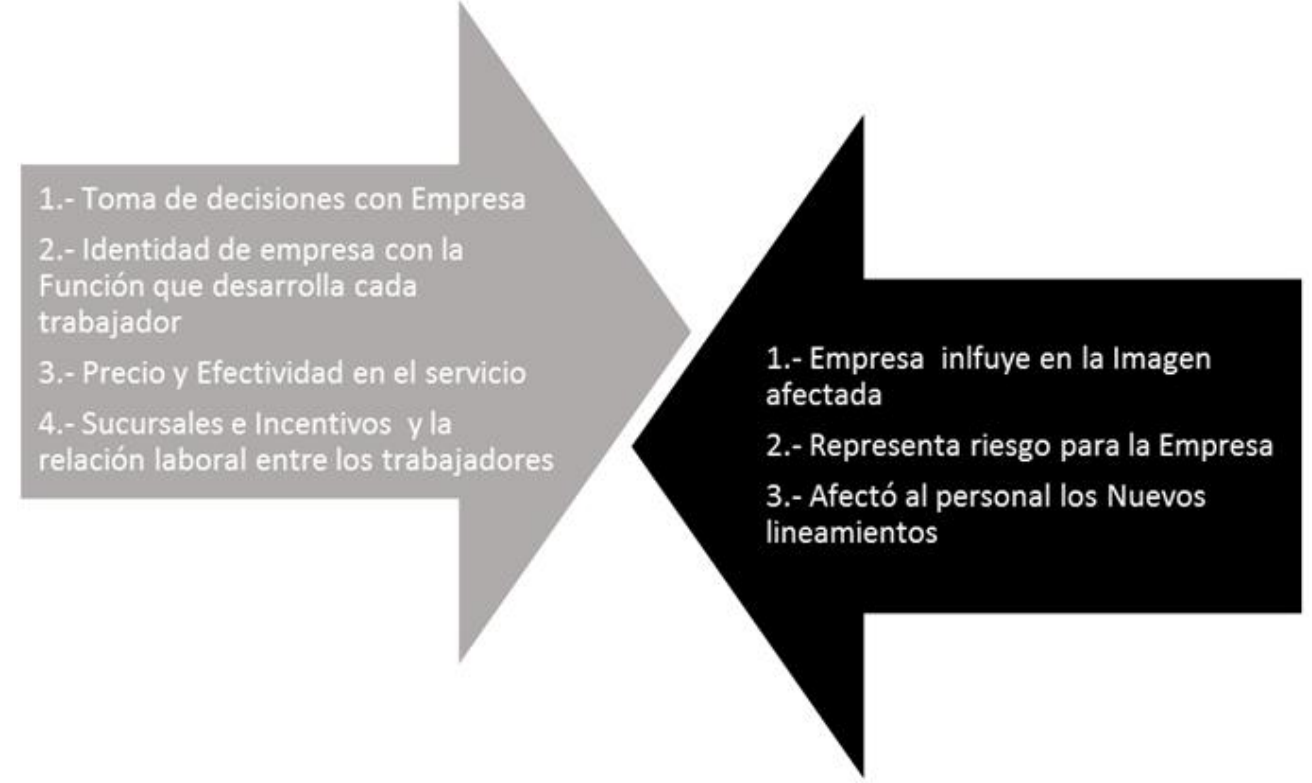

Fuente: Elaboración propia

\section{References:}

1. De Borja Solé Luis, Casanovas Pla Josep Andreu \& Bosh Campubí Ramón (2002). El Consumidor Turístico.

Madrid: Esic Editorial.

2. Govers Robert Go \& Frank M. (2004). Projected Destination Image on the World Wide Web: Content Analysis of Online Textual Representations of Dubai.

3. Hernández Mar Raúl (2015). Pueblos mágicos: discursos y realidades. México: Editorial Juan Pablos Editor. 
4. Kotler Philip \& Keller Kevin Lane (2008). Marketing Management. Pearson International Edition. 13th Edition.

5. Madrid Francisco (2014). Gobernanza Turística= destinos exitosos, El caso de los Pueblos Mágicos de México, México: Universidad Anáhuac México Norte.

6. Porter Michael, E. (2015). Estrategia Competitiva:Tecnicas para el analisis de los sectores. México. Grupo Editorial Patria. Segunda Ed.

7. Porter Michael, E. (2008). Las cinco fuerzas competitivas que le dan forma a la estrategia. Estados Unidos: Harvard Business Review.

8. OCDE (2013). Boletin cuatrimestral del Turismo $N^{\circ} 37$. México. OCDE

9. OCDE Estudios en Turismo. (2017) Es estudio de Política Turística de México. México

10. Segarra, C. (2014). Impacto estratégico de las fuerzas de Porter. México. D. F. Atenea Ediciones Académicas

11. Secretaría de Cultura y Turismo del Estado. DENUE 2017

12. SECTUR (2017). Monitoreo Data Tur. www.datatur.sectur.gob.mx (5 de septiembre de 2017).

13. SECTUR (2018). Turismo 2040: Política Turística de Estado. Memorias de los Foros Turismo es Bienestar. SECTUR

14. SECTUR (2019). Subsecretaría de Planeación y Política Turística. www.sectur.gob.mx (18 de mayo de 2019). 